\title{
Carnets
}

Revue électronique d'études françaises de l'APEF

Deuxième série - 1 | 2014

L'étranger

\section{L'étranger chez Montaigne : rejet et attirance}

\section{Maria Gloria Ríos Guardiola}

\section{(2) OpenEdition}

\section{Journals}

Édition électronique

URL : http://journals.openedition.org/carnets/1202

DOI : 10.4000/carnets. 1202

ISSN : 1646-7698

Éditeur

APEF

\section{Référence électronique}

Maria Gloria Ríos Guardiola, «L'étranger chez Montaigne : rejet et attirance », Carnets [En ligne], Deuxième série - 1 | 2014, mis en ligne le 30 mai 2014, consulté le 19 avril 2019. URL : http:// journals.openedition.org/carnets/1202; DOI : 10.4000/carnets.1202

Ce document a été généré automatiquement le 19 avril 2019.

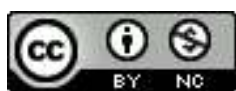

Carnets est mis à disposition selon les termes de la licence Creative Commons - Atribution - Pas d'utilisation commerciale 4.0 International. 


\title{
L'étranger chez Montaigne : rejet et attirance
}

\author{
Maria Gloria Ríos Guardiola
}

1 La notion d'étranger chez Michel de Montaigne est liée à la différence, à la distance, à l'altérité. Le parcours que je prétends suivre part de l'association de cette notion à deux mouvements opposés: d'une part le rejet, l'étonnement et même le dégoût que lui provoquent les circonstances politiques, religieuses et sociales de son pays, la colonisation et la civilisation européenne, et d'autre part l'attirance et la fascination de l'exemplarité du Nouveau Monde et de l'Antiquité, aussi bien que les moeurs d'autres pays, toujours associées à la curiosité et au désir d'exploration.

2 Montaigne se sent étranger au milieu d'une époque troublée, pleine de conflits. En raison de sa présence sur la scène politique et de son impartialité, il est requis pour affronter des situations extrêmement difficiles entre les deux factions religieuses -chrétienne et protestante - qui lui vaudront d'ailleurs son emprisonnement.

3 Épouvanté par les faits qui ont lieu en France (les luttes des princes pour l'accroissement de leur pouvoir, les querelles religieuses, la violence partout répandue, le danger couru à tout instant), il considère la plupart des idées ayant suscité ces événements comme étrangères, monstrueuses même. Particulièrement remarquables sont ses réflexions concernant la religion. Montaigne manifeste à maintes reprises son rejet des attitudes et des conduites des hommes au nom de celle-ci. D'après lui, il n'y a pas d'hostilité plus grande que la chrétienne :

Il n'est point d'hostilité excellente comme la chrestienne. Nostre zèle fait merveilles, quand il va secondant nostre pente vers la haine, la cruauté, l'ambition, l'avarice, la detraction, la rebellion.[...] Nostre religion est faicte pour extirper les vices : elle les couvre, les nourrit, les incite. (II, $12: 464$ ).

4 Mais à part la fonction morale et politique de la religion, les dogmatismes théologiques et l'hypocrisie religieuse, Montaigne manifeste son rejet vis-à-vis de beaucoup d'aspects propres à son contexte historique : la chasse aux sorcières entreprise par l'Église, par la Réforme ainsi que par la justice, le pouvoir de l'argent, l'état de la Justice (le rôle politique prépondérant des magistrats, la peine de mort, la torture judiciare, l'inégalité des 
hommes à l'égard de la Justice, ...), la persécution des Juifs par l'Inquisition et par le Portugal, ... À propos de chaque sujet problématique de son temps, Montaigne présente une vision différente, singulière, inusitée (Nackam, 1984:24) d'une perspective qui percevait comme étranger ce qui était normal aux yeux de ses contemporains.

En vue des bouleversements, son choix consiste alors à s'éloigner de la vie publique quitter ses charges, s'écarter de la vie politique, se retirer dans la tour de son château-, et à s'abstenir. Montaigne éprouve le besoin de se réserver un lieu à distance du monde où il puisse se faire spectateur de la vie des hommes et où il se sente libéré de tous les pièges. Cet exil physique, c'est le choix de la solitude et, pour Montaigne, il équivaut au choix de la liberté.

6 Cette relégation physique est accompagnée d'un exil idéologique attaché à l'écoute d'un passé qui le passionne : "Me trouvant inutile à ce siecle, je me rejecte à cet autre. Et en suis si embabouyné, que l'estat de ceste vieille Rome, libre, juste et florissante (car je n'en ayme, ny la naissance ny la vieillesse) m'interesse et me passionne. » (III, $9: 1043$ ).

7 Il se réfugie dans le passé glorieux des grandes civilisations -notamment romaine et grecque-, puisqu'il ne trouve pas sa place dans son siècle, de même que dans leurs langues. C'est la rencontre de ses idées avec celles des philosophes et des poètes de l'Antiquité. Fasciné, il ne peut alors résister la tentation de les citer et leurs paroles inondent particulièrement ses premiers essais car elles transmettent les valeurs intellectuelles et spirituelles de la pensée classique. En regard de cette invasion de paroles étrangères, qu'il admet, Montaigne se voit dans l'obligation de justifier l'emprunt comme un procédé parfaitement compatible avec l'invention personnelle. Il estime qu'il s'agit de l'assimilation des pièces empruntées pour les transformer et en faire un ouvrage personnel (II, X : 428).

8 En regard de ce passé glorieux, Montaigne situe la médiocrité de son époque et ce tempslà lui servira comme point de référence pour juger son présent (Burke, $1981: 17$; Glauser, 1972 : 96). En contestant de nouveau les usages de son temps, il révendique les valeurs que transmet le passé des anciennes civilisations :

Nos jugemens sont encore malades, et suyvent la depravation de nos moeurs: Je voy la pluspart des esprits de mon temps faire les ingenieux à obscurcir la gloire des belles et genereuses actions anciennes, leur donnant quelque interpretation vile, et leur controuvant des occasions et des causes vaines: Grande subtilité : Qu'on me donne l'action la plus excellente et pure, je m'en vois y fournir vraysemblablement cinquante vitieuses intentions.[...] La mesme peine, qu'on prent à detracter de ces grands noms, et la mesme licence, je la prendroye volontiers à leur prester quelque tour d'espaule pour les hausser. (I, $36: 235-236)$.

9 Cet exemple fait preuve de la relativité des perspectives et du procédé favori de Montaigne, celui d'inverser les rôles et de montrer ce qui nous paraît raisonnable, habituel et même juste, digne du plus grand rejet et viceversa.

10 À l'instar de ce qu'il fait dans l'exil de sa tour, Montaigne propose pour l'education des enfants l'étude de "ces grandes âmes des meilleurs siecles» (I, 25 : 162). Il préfère particulièrement les biographies -parmi lesquelles il distingue les Vies parallèles des hommes illustres de Plutarque ou Vie, doctrines et sentences des philosophes illustres de Diogène Laërce - pour nous rapprocher du côté plus humain des héros, de leurs attitudes plus que de leurs exploits. Nous pouvons y trouver, d'après Montaigne, tout un inventaire d'exemples à réexaminer sans cesse car ils révèlent les possibilités infinies de la nature 
humaine. En ce sens, l'Histoire, telle qu'il la conçoit, englobe l'anthropologie, en permettant la connaissance de l'autre et en acceptant ses différences (Boudou, 2001 : 55).

Il traite de plusieurs personnages illustres, modèles de vertu et exemples à suivre, parmi lesquels, il élit le général thébain Épaminondas, le roi macédonien Alexandre le Grand, les empereurs romains Jules César et Julien l'Apostat -ainsi nommé par la tradition chrétienne.

12 En parlant des empereurs romains, il s'attaque à la prodigalité des princes et montre le "prince idéal », en l'opposant au "tyran », tel qu'il est décrit par les théoriciens au XVI ${ }^{e}$ siècle. Montaigne révise spécialement des aspects biographiques de l'empereur Julien, que la littérature écclésiastique avait calomnié en raison de son rejet de la religion chrétienne. Cette attitude avait été renforcée par la Réforme, qui qualifiait de perfidie sa tolérance vis-à-vis de tous les cultes religieux (on sait qu'il essaya d'améliorer la situation des Juifs et qu'il s'engagea à reconstruire le temple de Jérusalem (Nackam, 1984: 361)). Montaigne arrive même à le comparer avec Henri II car il prend une décision semblable mais à différent propos: si Julien prétend la liberté de conscience, Henri III suscite des dissensions à l'intérieur du pays, ce qui est caractéristique du tyran, pour éviter une coalition contre lui. De même, Julien tranche sur les rois de Castille et de Portugal dans l'essai «Contre la fainéantise » (II, 21) : après avoir critiqué leur caractère belliqueux et leur manque de valeur en s'étant approprié les Indes par délégation, sans jamais avoir osé $\mathrm{y}$ aller après la conquête, Montaigne introduit la réflexion de Julien à propos des philosophes et des hommes de bien consacrés à des actions vertueuses:

Et cherchent autre adherent, que moy, ceux qui veulent nombrer entre les belliqueux et magnanimes conquerants, les Roys de Castille et de Portugal, de ce qu'à douze cent lieus de leur oisive demeure, par l'escorte de leurs facteurs, ils se sont rendus maistres des Indes d'une et d'autre part : desquelles c'est à sçavoir, s'ils auroyent seulement le courage d'aller jouyr en presence. L'Empereur Julian disoit encore plus, qu'un philosophe et un galant homme, ne devoient pas seulement respirer : c'est à dire, en donnez aux necessitez corporelles, que ce qu'on ne leur peut refuser; tenant tousjours l'ame et le corps embesongnez à choses belles, grandes et vertueuses (II, $21: 715$ ).

13 Julien s'erigea alors en symbole de toute l'école historique contemporaine de Montaigne en rétablissant la vérité faussée par l'Église-, devenant un personnage très attirant pour un intellectuel $d u \mathrm{xVI}^{\mathrm{e}}$ siècle à cause de son éducation chrétienne et ses croyances païennes.

De surcroît, les livres d'histoire offrent une grande variété de situations qui éveillent sa curiosité, certaines d'elles sont considérées par Montaigne comme des «inventions » en faisant porter l'accent sur son "étrangeté » (III, 6: 945). Un autre exemple que j'emprunte à l'essai «De la force de l'imagination » insiste sur ce même aspect : «Et aux diverses leçons, qu'ont souvent les histoires, je prens à me servir de celle qui est la plus rare et memorable. » (I, $20: 108)$

Son désir de connaître et d'explorer l'autre, lié à un certain exotisme, l'amène à s'intéresser aussi aux indigènes du Nouveau Monde. Montaigne peut être considéré comme l'un des premiers européens où jaillit la curiosité envers la réalité américaine. Au moment de commencer ses Essais, les grandes conquêtes coloniales étaient déjà achevées. Montaigne s'intéresse à toute sorte de récits de ces expéditions, à des documents ethnographiques ou à des engins indigènes (I, $30: 214$ ) pour découvrir et se familiariser avec ce nouveau continent et mener à bien cette étude d'anthropologie comparée. Parmi ceux-ci, il privilégie l'Histoire Générale des Indes de Francisco López de Gomara, secrétaire 
d' Hernán Cortés, et les témoignages oraux sur ce qui se passait dans le continent américain.

En rapport avec ses sources, nous pouvons différencier deux moments dans le traitement de ce sujet chez Montaigne. Dans une première étape, jusqu'à l'édition de 1580, les concepts introduits sont le résultat de la lecture des récits d'André Thévet (1558) à propos de l'expédition au Brésil de Villegagnon et de Jean de Léry, dont sa publication est le résultat d'un séjour risqué. Ce témoignage est d'accord avec son propre expérience lors de l'entretien à Rouen de Charles IX et trois indiens tupis en 1562, auquel il put assister (Núñez, 1997). En s'écartant de l'imagerie intarissable de fantaisie et de mystère qui regardait l'Amérique comme un produit de la barbarie et de l'impiété, Montaigne essaie d'envisager la réalité américaine à partir de son raisonnement humaniste.

Dans l'essai «Des Cannibales », Montaigne met l'accent sur l'aspect des moeurs qui choquait le plus violemment les Européens ; il essaie de montrer cette pratique socialisée, à finalité symbolique, loin de la bestialité attribuée aux « sauvages ». Une fois démontrée leur contingence, les lois et les coutumes en signifient plus que l'identité du groupe dont elles émanent ; cela suffit pour les respecter (Tournon, 1989 : 97).

Montaigne s'interroge sur la condition de l'étranger dans cet essai et arrive à nous faire penser à l'étrangeté qui enferme ce qui est proche: Qui est "barbare»? Qui est le "sauvage »? Le texte effectue une opération de redistribution de l'espace culturel dont l'effet est de fixer ou de déplacer les frontières qui délimitent les domaines culturels (le familier vs l'étranger) en arrachant ces expressions à l'usage ordinaire que l'on en fait (Höffe, 2007 : 269). Il se produit un glissement sémantique où le mot «sauvage » dérive vers le « naturel » et s'oppose soit à l'artifice qui altère la nature, soit à la frivolité. Il va de soi qu'il s'agit en tout cas d'une connotation positive. En revanche, le mot «barbare » revient en Europe, au même lieu d'où il venait, attribué à la civilisation qui l'excluait :

Je pense qu'il y a plus de barbarie à manger un homme vivant, qu'à le manger mort, à deschirer par tourmens et par gehennes, un corps encore plein de sentiment, le faire rostir par le menu, le faire mordre et meurtrir aux chiens, et aux pourceaux (comme nous l'avons non seulement leu, mais veu de fresche memoire, non entre des ennemis anciens, mais entre des voisins et concitoyens, et qui pis est, sous pretexte de pieté et de religion) que de le rostir et manger après qu'il est trespassé. (I, $30: 216)$

Dans la récréation de ce monde, Montaigne réproduit une version de l'« Âge Dorée ", époque où l'homme profitait de son loisir, sans propriétés, sans lois, sans État, dans un éternel printemps (Jaúregui, 2008 : 187), en accord avec une nouvelle tendance qui surgit en Europe et qui considère les coutumes des indigènes produit de l'innocence de l'homme, que l'on croyait à tout jamais perdue. L'exemple suivant caractérise positivement la société indigène au moyen de la négation de ce que l'on peut attribuer à la civilisation européenne :

C'est une nation, diroy-je à Platon, en laquelle il n'y a aucune espece de trafique ; nulle cognoissance de lettres ; nulle science de nombres ; nul nom de magistrat, ny de superiorité politique; nul usage de service, de richesse, ou de pauvreté ; nuls contrats; nulles successions; nuls partages; nuls occupations, qu'oysives; nul respect de parenté, que commun; nuls vestements ; nulle agriculture ; nul metal ; nul usage de vin ou de bled. Les paroles mesmes, qui signifient la mensonge, la trahison, la dissimulation, l'avarice, l'envie, la detraction, le pardon, inouyes. Combien trouverait il la republique qu'il a imaginée, esloignée de cette perfection? (I, $30: 212-213)$ 
d'autres sources plus véraces. Dans l'essai « Des coches » (III, 6), Montaigne nous parle d'un monde entièrement différent du nôtre, c'est-à-dire de la civilisation européenne, qui est malade et qui a contaminé ce monde « si nouveau et si enfant ».

21 Il y distingue aussi la réalité du Pérou, du Brésil et du Mexique. Il est non seulement motivé par la curiosité ou son penchant pour le pittoresque, mais encore il prend parti pour les Indiens et il s'en prend à la brutalité des conquérants de l'Amérique, en protestant contre les traitements inhumains infligés à ces gens.

À cette fin, il utilise souvent des métaphores et des comparaisons pour mettre en relief ce qu'il dénomme domination au lieu de colonisation. Son propos est de désarticuler tous les arguments présentés qui prétendent justifier l'imposture et la cruauté avec laquelle ils sont traités, de même que l'excès et la lâcheté que suppose l'utilisation de moyens puissants et sophistiqués vis-à-vis du primitivisme des indigènes.

Ce n'est pas à dire pour autant qu'il n'éprouve que de l'attirance envers les Indiens, dans l'essai « De la modération » $(\mathrm{I}, 29)$ - où l'on décrit un sacrifice mexica- nous trouvons un autre regard de Montaigne. Il y reprend la notion de cannibalisme, mais sa perspective diffère selon son propos : le tupi et le mexica sont des cannibales mais seul le dernier est un cruel sauvage. Alors que le tupi est vertueux, guerrier, masculin et naturel, le mexica est corrompu, artificiel, cruel et "civilicé », en le rapprochant de sa perception de l'européen. Il faudrait donc remarquer que Montaigne se débat entre la fascination et l'horreur en ce qui touche au sauvage et, à différence de Rousseau qui prenait la société primitive des indigènes comme exemple de perfection, il s'en sert pour mettre en évidence le désordre et la corruption des sociétés de son temps (Bakewell, 2011 : 237).

Cette séduction que Montaigne éprouve envers l'étranger va se concrétiser dans le seul voyage qu'il entreprit en Italie, en passant par la Suisse et l'Allemagne, qui lui permit de s'éloigner d'une part de ses propres devoirs et d'autre part du déchirement que la France subissait («L'autre cause qui me convie à ces promenades, c'est la disconvenance aux moeurs presentes de nostre estat. » (E. III, $9: 1000)$.

Il partit pendant dix-sept mois à la découverte d'autres pays, tel que son Journal de voyage nous décrit. Montaigne y réfléchit aussi au concept de voyage, en rapprochant celui-ci au plaisir du mouvement et de l'exploration des lieux inconnus :

il n'allait, quant à lui, en nul lieu que là où il se trouvait, et qu'il ne pouvait faillir ni tordre sa voie, n'ayant nul projet que de se promener par les lieux inconnus, (J. ${ }^{1}$ : 197).

Il perçoit son environnement uni à la routine (« ces ordinaires goutières me mangent et m'ulcèrent. Les inconvenients ordinaires [...] sont continuels et irreparables ", E. III, 9 : 995), à la coutume, à la monotonie, à la lassitude, tandis que le voyage fournit l'accès au nouveau et il est lié à la mobilité, à l'éloignement, au changement, à la variété et au plaisir. Son attitude contraste avec celle qu'adoptent ceux qui se réjouissent quand ils rencontrent quelqu'un de leur pays au cour de leur voyage, craignant d'être contaminés par l'inconnu et désirant rentrer parce qu'il n'y a rien de mieux que leur pays :

Quant j'ay esté ailleurs qu'en France : et que, pour me faire courtoisie, on m'a demandé, si je vouloy estre servi à la Françoise, je n'en suis mocqué, et me suis tousjours jetté aux tables les plus espesses d'estrangers. J'ay honte de voir nos hommes enyvrez de cette sotte humeur, de s'effaroucher des formes contraires aux leurs [...] Où qu'ils aillent, ils se tiennent à leurs façons et abominent les estrangeres. Retrouvent ils un compatriote en Hongrie, ils festoyent cette aventure 
et voylà à se rallier et à se recoudre ensemble, à condamner tant de moeurs

barbares qu'ils voient (E. III, $9: 1031$ ). avide des choses nouvelles et inconnues ayde bien à nourrir en moi le désire de voyager "; et un peu plus tard admet que cette voracité est telle qu'il en arrive au point de vouloir se sentir satisfait malgré le grand plaisir qu'il éprouve : 
La diversité des façons d'une nation à autre ne me touche que par le plaisir de la variété. Chaque usage a sa raison. Doyent des assiettes d'estain, de bois, de terre, bouilly ou rosty, beurre ou huyle de nois ou d'olive, chaud ou froit, tout m'est un, et si un que, vieillissant, j'accuse cette genereuse faculté, et auroy besoin que la delicatesse et le chois arrestat l'indiscretion de mon appetit et parfois soulageat mon estomac (E.III, $9: 1031$ ). secrétaire conjecture dans le journal que si Montaigne n'avait pas commencé le parcours accompagné d'autres gentilshommes, il se serait dirigé vers la Cracovie ou la Grèce (J. : 153), au lieu d'aller à Rome qu'il ne connaissait pas personnellement mais par l'intermédiaire de nombreux Français. On ne manquera pas d'observer que cette indifférence de Montaigne envers la ville, objectif des humanistes et des amoureux de la culture classique entre en contradiction avec sa présence récurrente dans ses Essais. Cela est dû d'un côté aussi bien à son désir de nouveauté et de découverte qu'au refus des lieux communs et la probable rencontre d'un grand nombre de Français (J. : 160, 189), d'un autre côté Montaigne perçoit la métropole de Rome comme un grand tombeau de la gloire passée (J. : 200-201). Il ne la reconnaît pas comme la ville « abstraite et contemplative » du passé qu'il revendique comme patrie intellectuelle (Tournon, 1989:237). Ce qu'il trouve en arrivant à Rome, c'est une ville de faste et de cérémonies où la censure s'exerce de manière implacable (J. : 191).

En contrepartie, Montaigne éprouve l'attirance de la beauté de cette ville, qui surpasse celle de Paris « en nombre et grandeur des places publiques, beautés des rues, et beautés des maisons » (J. : 191), de même que son universalité, son hétérogénéité et son accueil de l'homme indépendamment de son lieu d'origine.

Il est intéressant de souligner son désir et sa capacité d'adaptation aux moeurs locales quand il établit sa résidence près des Bains della Villa. En mettons comme exemple la fête paysanne qu'il organise conformément aux usages de la région (J. : 286). Force est de dire que l'on assiste alors à un changement de perspective, en passant de l'observation -qui lui est propre- à l'interaction, mouvement qui entraîne également la perte de sa condition d'étranger. Montaigne célèbre l'acceptation et l'accueil reçus d'une population préoccupée de ses labeurs quotidiens, privée du temps pour fréquenter des étrangers. À partir de cet événement, il sent qu'il n'est plus perçu comme un voyageur et même qu'il 
est fort apprécié ( J'y reçus de tout le monde le meilleur accueil et des caresses infinies », $\mathrm{J} .:$ p. 327). Nous pouvons donc observer que l'on passe de l'adaptation comme tolérance à l'adaptation comme assimilation et expérience vécue.

Encore qu'il se vante dans ses essais de faire une immersion dans les moeurs du lieu visité, dans son Journal de voyage nous trouvons de nombreuses références aux incommodités telles que l'absence de contrevents, de rideaux autour du lit (J.:138) ou dans les fenêtres (J.: 178), à propos de la literie, des toilettes ou concernant des usages tels que celui de la table en Suisse auquel son secrétaire fait allusion :

M. de Montaigne, pour essayer tout à fait la diversité des moeurs et façons, se laissait partout servir à la mode de chaque pays, quelque difficulté qu'il y trouvât. Toutefois en Suisse il disait qu'il n'en souffrait nulle que de n'avoir à table qu'un petit drapeau d'un demi-pied de long pour serviette (J. :101).

Ou lui-même dans ses Essais : «Je disnerois sans nape : mais à l'Alemande, sans serviette blanche, très-incommodéement. Je les souille plus qu'eux et les Italiens ne font : et m'aide peu de cullier, et de fourchete. » (III, 13 : 1131-1132).

41 Je voudrais d'autre part faire remarquer son ouverture d'esprit dans son rapport et sa communication avec les autres. Il est un grand interlocuteur, grâce à quoi Montaigne est souvent invité au long de son voyage. Cela va de pair avec ses manières agréables qui encouragent les gens à demander son avis, comme il arrive aux usagers des Bains della Villa (J. : 297).

$42 \mathrm{Au}$ demeurant, l'attirance qu'éprouve Montaigne envers l'étranger se manifeste principalement dans trois axes -l'intérêt pour l'Antiquité, la curiosité pour les civilisations indiennes d'Amérique et son voyage en Italie- qui conduisent la pensée vers les lieux les plus éloignés dans le temps et dans l'espace.

43 À part l'attirance basée sur sa curiosité et son désir d'exploration, normalement en rapport avec l'aspect exotique ou anthropologique, Montaigne est porté à soutenir le parti des faibles, «étrangers » à sa classe sociale. Ce penchant s'étend aux animaux, aux cannibales d'Amérique, aux paysans et « aux pauvres gens ", « à l'enfant que tourmente le pédant, à tous ceux que l'on méprise ou que l'on opprime au nom d'une connaissance outrecuidante » (Starobinski, 1982 : 299), sans pour autant s'en tenir à la compassion pour ces êtres « inférieurs ", auxquels il reconnaît leur supériorité morale et intellectuelle dans certains domaines : d'une part ils sont lucides à l'heure d'apercevoir les abus de la société européenne -comme ils manifestent lors de leur entretien avec Charles IX à Rouen, décrit dans l'essai « Des cannibales » (I, $30: 220-221)^{2}$; d'autre part, ils sont plus humains jusque dans leurs guerres, quand il parle des cannibales (I, $30: 217)^{3}$.

Il est intéressant de remarquer la fréquente inversion de rôles que Montaigne effectue, en attribuant des rôles positifs à ceux qui en ont négatifs, à l'avis de ses contemporains, et au contraire. Ce procédé a dû provoquer le désarroi dans son temps car il change la perspective et la perception de son lecteur: le «monstre» dans ses essais n'est pas l'hérétique mais la cruauté de leurs bourreaux; « barbare » est la xénophobie et non pas l'étranger; "sauvage» est la persécution et non pas celui qui est persécuté; «monstrueuse » est l'inhumanité avide des princes de Portugal et non pas les Juifs dans leur détresse ; la guerre civile et non pas la Réforme ; «cruels » sont ceux qui pratiquent la torture sur ceux qui sont en vie et non pas les anthropophagues qui agissent sur les morts. 

Montaigne la notion d'étranger qui provoque son attirance se matérialise en deux mouvements: l'un vers l'éxtérieur, vers la différence, et l'autre vers l'intérieur, ce deuxième correspondant à son exil physique éloigné de sa réalité et marqué par son refuge dans le passé à travers les livres. Ces deux orientations revêtent sa propre perception d'étranger étant donné qu'il ne se sent pas faire partie de la société française de cette période et préfère se situer à l'écart de ce qui se passe. Ce sentiment est à l'origine d'un troisième mouvement qui relie l'idée d'étranger au refus qu'il éprouve face aux événements, croyances, lois et moeurs de son temps et qui le portent à dénoncer sa fausseté et sa corruption.

\section{BIBLIOGRAPHIE}

BAKEWELL, Sarah (2011). Cómo vivir o una vida con Montaigne. En una pregunta y veinte intentos de respuesta. Barcelona : Ariel.

BURKE, Peter (1981). Montaigne. Madrid : Alianza Editorial.

Boudou, Bénédicte (2001). Essais. Michel de Montaigne. Paris : Hatier.

GLAUSER, Alfred (1972). Montaigne paradoxal. Paris : Nizet.

HÖFFE, Otfried (2007). Ciudadano económico, ciudadano del Estado, ciudadano del mundo. Ética política en la era de la globalización. Madrid : Katz editores.

JAÚREGUI, Carlos A (2008). Canibalia : canibalismo, calibanismo, antropofagia cultural y consumo en América Latina. Madrid/ Frankfurt : Iberoamericana/ Vervuert.

Montaigne, Michel de (1962) : CEuvres complètes. Paris : Gallimard. «Bibliothèque de la Pléiade ».

MontAigne, Michel de (1983). Journal de voyage. Paris : Gallimard.

NACKAM, Géralde (1984). Les Essais de Montaigne. Miroir et procès de leur temps. Paris : Nizet.

NÚÑEZ, Estuardo (1997). Las letras de Francia y el Perú : apuntaciones de literatura comparada. Lima : Universidad Nacional Mayor de S. Marcos.

PRADO, Javier del (1997-1998). «Montaigne : écriture et itinérance », Cuadernos de Filología Francesa , no10, pp. 35-53.

STAROBINSKI, Jean (1982). Montaigne en mouvement. Paris : Gallimard.

TouRnON, André (1989). Montaigne en toutes lettres. Paris: Bordas. 


\section{NOTES}

1. Cette abréviation fait allusion à son journal de voyage.

2. Ils trouvaient fort étrange la soumission de tant de grands hommes à un roi enfant ainsi que l'existence d'hommes riches et des mendiants et l'acceptation ou non rébellion de ceux derniers.

3. Leurs guerres ne sont pas motivées par l'acroissement des territoires ou des richesses mais par l'obtention du privilège d'être supérieur en valeur.

\section{RÉSUMÉS}

Le concept d'étranger chez Montaigne peut se concrétiser en deux axes pouvant être associés aux notions de rejet et d'attirance : d'un côté l'exil ideólogique et physique choisi par cet auteur, et de l'autre l'appel de l'altérité, lié au désir d'exploration. Il se sent étranger au milieu d'une époque troublée. Il considère les idées ayant suscité ces conflits comme étrangères, monstrueuses même. Son choix consiste alors non seulement à quitter ses charges et à se retirer dans la tour de son château, mais aussi à se réfugier dans le passé glorieux des grandes civilisations -notamment romaine et grecque- et dans leurs langues qu'il privilégie pour les nombreuses citations de ses Essais. Cette position de rejet forme un net contraste avec son attirance pour l'étranger en tant que différent, inconnu et même méconnu. Son désir de connaître et d'explorer l'autre, l'amène à s'intéresser aux indigènes du Nouveau Monde ou à partir à la découverte d'autres pays.

The concept of étranger in Montaigne may come in two axis associated with the notions of rejection and attraction: on the one hand, the ideological and physical exile chosen, and, on the other, a foreign fascination linked to the desire for exploration. He feels himself strange in the middle of a troubled period. He considers the ideas that have provoked these conflicts odd, even grotesque. Therefore, his choice is not only to give up his public responsabilities and retire in the tower of his castle, but also to find out a shelter in the glorious past of the great civilizations, especially Roman and Greek ones, and in their languages, highly used in a huge amount of quotations from his essays. This position of rejection contrasts with his attraction towards the stranger, just for being different, unknown, even unrecognized. His desire to learn and explore the other takes himself to get into through the New World natives or to get into contact with other countries to be discovered.

\section{INDEX}

Keywords : Montaigne (Michel de), attraction, rejection, stranger, foreigner, outsider, New World

Mots-clés : Montaigne (Michel de), attirance, rejet, étranger, Nouveau Monde 
AUTEUR

MARIA GLORIA RÍOS GUARDIOLA

Universidad de Murcia

gloriarg[at]um.es 